\title{
The Correlation of Carbon Monoxide Level, Lactate, Creatine Kinase Myocardial Band, Troponin T, Magnetic Resonance Imaging and Clinical Results in Acute Carbon Monoxide Poisoning
}

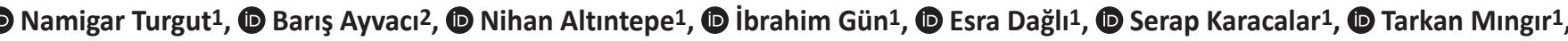 \\ (D) Hatice Feyizi ${ }^{1}$, (D) Yaşar İncekaya ${ }^{1}$, (1D Suzan Deniz Önol ${ }^{3}$ \\ 1 University of Health Sciences Turkey, İstanbul Okmeydanı Training and Research Hospital, Clinic of Anesthesiology and Intensive Care, Istanbul, Turkey \\ 2 University of Health Sciences Turkey, Istanbul Okmeydanı Training and Research Hospital, Clinic of Emergency Medicine, Istanbul, Turkey \\ 3 University of Health Sciences Turkey, İstanbul Okmeydanı Training and Research Hospital, Clinic of Radiology, Istanbul, Turkey
}

\section{Abstract}

Objective: In this study, it was aimed to investigate the correlation between arrival-carboxyl hemoglobin (COHb) levels, lactate, creatine kinase-myocardial band (CK-MB), troponin $\mathrm{T}$ and radiological findings in patients who were admitted to emergency service with a prediagnosis of carbon monoxide (CO) poisoning between the years 2014-2016.

Methods: The patients who were admitted to emergency service with a pre-diagnosis of CO poisoning between the years 2014-2016 were screened retrospectively. Demographic data, arrival-COHb levels, lactate, CK-MB, troponin T, and if available, computed tomography or magnetic resonance (MR) imaging of the patients, their clinical treatment, and results were compared.

Results: While $\mathrm{COHb}$ values of the patients at emergency department arrival were $14.536 \pm 13.047 \%$, they were found as $4.536 \pm 3.698 \%$ at discharge from the hospital. A correlation was not observed statistically between arrival-COHb, lactate, troponin T, and CK-MB. Arrival-lactate level was found as $>2.1 \mathrm{mmol} / \mathrm{L}$ in 47 patients (36.90\%). Cranial MR findings that suggest CO poisoning were determined in four cases who underwent hyperbaric oxygen treatment; millimetric signal enhancements being concordant with chronic microangiopathic ischemic gliotic foci in white matter were determined in the level of the periventricular field, bilateral corona radiata and centrum semiovale in MRI.

Conclusion: There is no correlation between arrival- $\mathrm{COHb}$ levels of the patients and lactate, CK-MB and troponin $\mathrm{T}$ in $\mathrm{CO}$ poisoning. Radiological evaluation should be used for excluding the reasons for a neurological disorder, not for diagnostic reasons.

Keywords: Carbon monoxide poisoning, lactate, troponin, creatine kinase

\section{INTRODUCTION}

Carbon monoxide (CO) is a gas that is produced through incomplete combustion of fuels that contain "carbon" and it is regarded as highly "toxic". CO poisoning is the most frequently encountered poisoning in Turkey (1).

In case of breathing, CO causes tissue hypoxia by two important effects: First, the binding capacity of CO to hemoglobin is 200-300 times more than that of oxygen, and second, the part dissolved in plasma causes decrease in 2,3-diphosphoglyceride production thus leading to left shift in oxyhemoglobin dissociation curve, and the oxygen that is bound to hemoglobin cannot be released. Because of the first effect, oxygen content of blood decreases, and because of "carboxyhemoglobin $(\mathrm{COHb})$ " produced by the second effect, oxygen offer to tissues decreases. Therefore hypoxia develops in tissues, and then anaerobic glycolysis increases, thus leading to lactic acidosis; 
the formation of free oxygen radicals, hypotension, and loss of consciousness are followed by lipid peroxidation and apoptosis (2). Multi-variance analyses suggested that arrival-lactate level, old age, leucocyte, and mental condition are independent factors related to critical complications and need of intensive medical treatment in order to determine the patient prognosis after CO poisoning (3). Nevertheless, the medical records on $\mathrm{CO}$ poisoning cases in our country are considered to be inadequate. Also, the number of patients applied to health institutions because of $\mathrm{CO}$ poisoning and how many of them were diagnosed with $\mathrm{CO}$ poisoning are unknown; moreover, the diagnoses are considered to be recorded with different codes in ICD-10 coding system (4).

In this study, it was aimed to investigate the correlation between arrival- $\mathrm{COHb}$ levels and lactate, creatine kinasemyocardial band (CK-MB), troponin $\mathrm{T}$, and radiological findings of the patients who were admitted to emergency service with a pre-diagnosis of CO poisoning between the years 2014 and 2016

\section{METHODS}

\section{Subjects and Study Protocol}

Following the approval of the Ethics Committee of Ministry of Health Okmeydanı Training and Research Hospital (approval date: 14/06/2016, decision no: 495), the patients admitted to emergency service with a pre-diagnosis of $\mathrm{CO}$ poisoning between the years 2014 and 2016 were screened retrospectively.

\section{Measurements and Calculations}

Demographic data, arrival-COHb levels, lactate, CK, troponin $\mathrm{T}$, and, if available, brain computed tomography (CT) scans or magnetic resonance imaging (MRI) of the patients, their clinical treatment and results were compared.

\section{Statistical Analysis}

Statistical analysis of the data was performed by SPSS 21.0 (SPSS, Chicago, IL). The correlation between arrival- $\mathrm{COHb}$ and lactate, troponin T, CK-MB was analyzed by the Pearson correlation test. $P$ values of $<0.05$ were considered significant.

\section{RESULTS}

While $\mathrm{COHb}$ values of the patients at emergency department arrival were $14.536 \pm 13.047 \%$, they were found as $4.536 \pm 3.698 \%$ at discharge from the hospital (Table 1). A correlation was not observed statistically between arrival-COHb, lactate, troponin $\mathrm{T}$ and CK-MB (Table 2, Figure 1, $\mathrm{p}>0.05$ ). Arrival-lactate level was found as $>2.1 \mathrm{mmol} / \mathrm{L}$ in 47 patients (36.90\%). Cranial MRI findings that suggest CO positioning were determined in four cases who underwent hyperbaric oxygen (HBO) treatment; millimetric signal enhancements being concordant with chronic microangiopathic ischemic gliotic foci in white matter were determined in the level of the periventricular field, bilateral corona radiata and centrum semiovale in MRI (Figure 2).

\section{DISCUSSION}

The first symptoms of $\mathrm{CO}$ intoxication are nonspecific; history is the most valuable finding. Physical examination has been used to a limited extent in diagnosis. Blood $\mathrm{COHb}$ levels should be measured as soon as possible; nevertheless, $\mathrm{COHb}$ levels frequently may not be useful in diagnosis and treatment (halflife is 4-6 hours at room air) (5). The relationship between the severity of poisoning and blood CO level is not drastic, but it can be used as an indicator to follow-up on the treatment. On

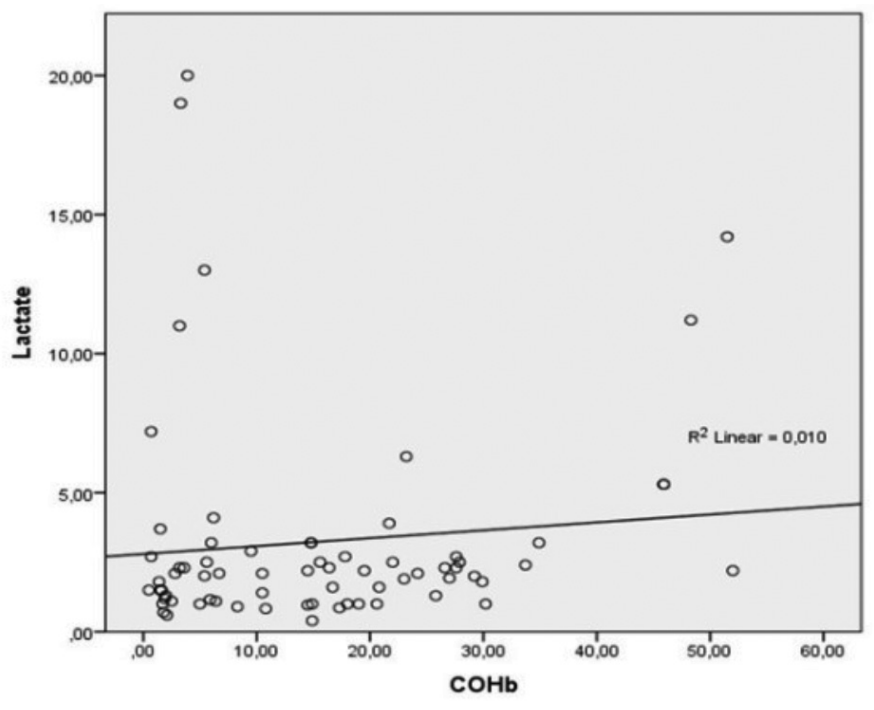

Figure 1. Correlation between arrival- $\mathrm{COHb}$ and lactate $\mathrm{COHb}$ : Carboxyl hemoglobin

Table 1. Carboxyl hemoglobin, lactate, troponin T, creatine kinase-myocardial band levels of the cases on admission to the emergency department

\begin{tabular}{|l|l|l|l|l|l|}
\hline & Mean \pm SD & $\mathbf{n = 1 3 2}$ & & \multicolumn{5}{|l|}{} \\
\hline Arrival COHb (\%) & Discharge COHb (\%) & Lactate $(\mathbf{m m o l} / \mathrm{L})$ & Troponin T & CK-MB & Length of hospital stay \\
\hline $14.536 \pm 13.047$ & $4.536 \pm 3.698$ & $3.172 \pm 3.670$ & $0.086 \pm 0.478$ & $3.226 \pm 6.849$ & $1.410 \pm 1.668$ \\
\hline COHb: Carboxyl hemoglobin, CK-MB: Creatine kinase-myocardial band, SD: Standard deviation
\end{tabular}


the other hand, if a long time passed after $\mathrm{CO}$ exposure or if supportive oxygen treatment was administered, blood CO level can erroneously be detected as low. Detection of high-level CO in the blood is important for diagnosis, but low CO level in blood does not detract from diagnosis (6). In the early period or weeks after the exposition, various symptoms occur in organs because of hypoxia. In addition to major symptoms affecting the neuropsychiatric and cardiovascular systems, other systems and organs are also affected (7-9). Especially when the children with acute $\mathrm{CO}$ poisoning were compared with those who were healthy, the heart seems to be the most critical organ and subclinical systolic and diastolic left ventricle dysfunction, and ventricular repolarization failure were observed (10). Similar findings ranging from simple arrhythmia to myocardial infarction are also seen in the poisoning of adults (11). Thus, a correlation is expected between blood $\mathrm{COHb}$ and cardiac troponin $\mathrm{T}$. In their

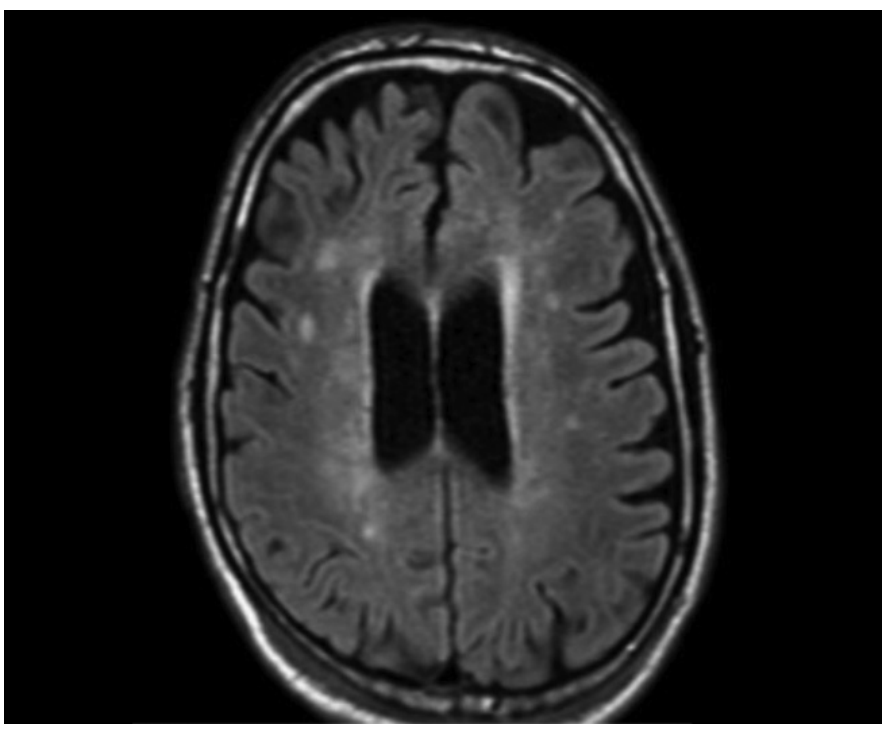

Figure 2. Cranial MRI; millimetric signal enhancements being concordant with chronic microangiopathic ischemic gliotic foci in white matter were determined in the level of the periventricular field, bilateral corona radiata and centrum semiovale in MRI MRI: Magnetic resonance imaging retrospective study in 141 patients, Huysal et al. (1) found a weak correlation for this, although the $\mathrm{COHb}$ level greater than $25 \%$ was described as severe acute CO intoxication and, they found no correlation with CK-MB. Also, in our study, a correlation was not found between arrival-COHb (14.536 \pm 13.047$)$ and troponin $\mathrm{T}$ and CK-MB. In another study on correlation from the point of arrival-blood lactate, $\mathrm{COHb}$, and electrocardiography (ECG) findings ( $\mathrm{COHb}$ values on average $21.5 \pm 13.9 \%)$, normobaric oxygen treatment was applied to $67.6 \%$ of the patients while HBO treatment was administered to $32.4 \%$ of the patients. Blood lactate levels of HBO treated patients were found greater when admitted to emergency service $(2.3 \mathrm{mmol} / \mathrm{L}$ vs $1.0 \mathrm{mmol} / \mathrm{L}$, $\mathrm{p}<0.001)$, and a positive correlation was determined between lactate level and clinical findings of poisoning and $\mathrm{COHb}$ values. Nevertheless, this study was criticized on the basis of its results, such that HBO treatment is a therapy method rather than a measure of clinical outcome, and more than one factor is required for the decision regarding HBO therapy, and it does not indicate more severe poisoning (12). Also, in our study, although arrival-lactate values of the patients were above 3, no correlation was found between arrival- $\mathrm{COHb}$ and arrival-lactate values. Arrival-COHb values above $30 \%$, low lactate, troponin T, and Glasgow Coma scale (GCS) show that more than one factor was considered in the decision-making process for four patients whose HBO treatments were planned. In another study, HBO treatment was planned for 37 of 57 patients who admitted to the emergency department for $\mathrm{CO}$ poisoning (COHb levels $10.1 \pm 5.7 \%$ (range $=3-25 \%$ ) (13). HBO treatment was administered to four patients in our study. The first patient had a GCS score of 4 in the neurologic examination, was hypotensive, had metabolic acidosis in arterial blood gas, $\mathrm{COHb}$ level was 30\%, and lactate was $2.2 \mathrm{mmol} / \mathrm{L}$, ECG showed dysrhythmia, ST-T variances and had pulmonary edema. The second patient had a $\mathrm{COHb}$ level of $52 \%$, lactate was $2.2 \mathrm{mmol} / \mathrm{L}$. The third patient had a $\mathrm{COHb}$ level of $51 \%$ and lactate was $14.2 \mathrm{mmol} / \mathrm{L}$. The fourth patient

Table 2. Correlation of arrival- carboxyl hemoglobin, lactate, troponin T, creatine kinase-myocardial band

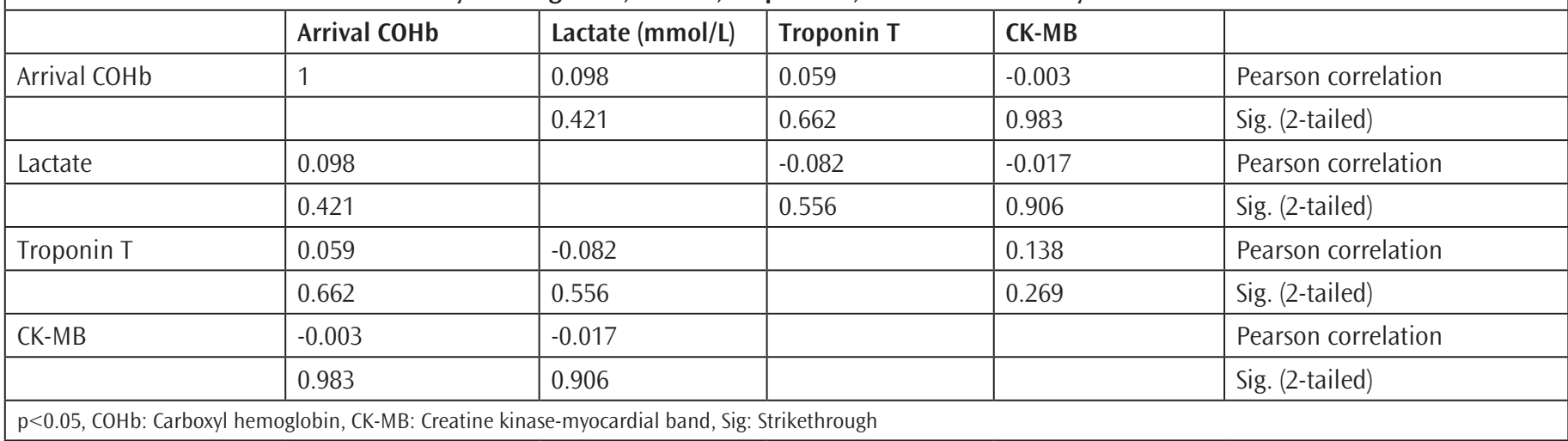


had a $\mathrm{COHb}$ level of $45.9 \%$ and lactate was $5.3 \mathrm{mmol} / \mathrm{L}$. The normal level of $\mathrm{COHb}$ is $0.5-3 \%$, and it is $3-7 \%$ in neonatal and $4-12 \%$ in smokers. Poisoning begins when the concentration is $15 \%$. The toxic level is $20-50 \%$ and the lethal level is above $50-60 \%$. $\mathrm{COHb}$ level was found $50 \%$ in people staying several hours in an environment with CO, and it was found as $25 \%$ in a person staying in such an environment for 34 minutes (14). Mild poisoning can have subtle symptoms such as headache, fatigue, weakness, gasping, nausea, and vertigo ( $\mathrm{COH} b=15-30 \%)$. When the level of $\mathrm{COHb}$ exceeds $20 \%$, the heart and brain are affected severely, and if it is above $30-70 \%$, it leads to dizziness, vomiting, loss of muscular coordination, unconsciousness and death (15).

The first treatment option in CO poisoning is $100 \% \mathrm{O}_{2}$ (normobaric oxygen) treatment at least for six hours until reaching the normal values of $\mathrm{COHb}$. The aim is to decrease $\mathrm{COHb}$. Continuous oxygen treatment should be carried on until the patient becomes asymptomatic, or the level of $\mathrm{COHb}$ is below $10 \%$. It should be decreased to $2 \%$ in those with cardiovascular or pulmonary symptoms (16).

HBO modulates the inflammatory processes that cannot be maintained with normobaric oxygen, enhances mitochondrial function, and temporarily inhibits lipid peroxidation. It also repairs leukocyte adhesion to damaged microvasculature and promotes myelin formation in the brain. Therefore, HBO treatment is recommended in acute symptomatic CO poisoning and, in the same way, in poisoning due to CO inhalation for patients with unconsciousness and those with the permanent neurological deficit; nevertheless, it remains somehow unclear $(17,18)$. Brain CT or MRI should be used for excluding the reasons for a neurological disorder, not for diagnostic reasons. Hypoxic brain damage is primarily observed in the cerebral cortex, cerebral white matter, and basal ganglions, especially in Globus pallidus (19). In our study, cranial MRI findings that suggest CO positioning were determined in four cases; millimetric signal enhancements being concordant with chronic microangiopathic ischemic gliotic foci in white matter were determined in the level of the periventricular field, bilateral corona radiata, and centrum semiovale.

A standard HBO treatment protocol for $\mathrm{CO}$ poisoning includes $100 \%$ oxygen for 90 minutes. Cases with moderate symptoms can benefit from the initial treatment. However, for the patients whose symptoms do not regress, second or third treatment sessions could be planned. Temporary unconsciousness, coma or seizure, ischemic ECG changes, focal neurological deficit, pregnant women with $\mathrm{COHb}$ levels $>15 \%$, $\mathrm{COHb}$ levels exceeding $40 \%$ together with headache and nausea are the indications for HBO treatment. The only absolute contraindication for HBO treatment is untreated pneumothorax (20). In a study involving 68 centers from 23 countries, temporary or prolonged unconsciousness was accepted as an indication for HBO treatment in 95\% of the centers; also, positive neurologic findings, acute cardiac ischemia and, pregnancy were of priority for HBO treatment (21). Our priorities were formed by these data in our four cases; for our first case, the symptoms regressed after the first session, and therefore, the treatment program was terminated, nevertheless for our second case, there was no regression even after three sessions. In their ten years of study, Chan et al. (22) administered HBO treatment for 24 of 93 patients, and none of these patients were found to have neurological sequelae in the follow-up. Neurological sequelae were found in seven patients who were not treated with HBO. The degree of the patient's consciousness on admission to the hospital, GCS score, increased troponin levels, creatine kinase, and intubation requirement were defined as prognostic factors for the development of neurological sequelae $(21,23,24)$.

\section{CONCLUSION}

There was no correlation between arrival- $\mathrm{COHb}$ levels of the patients and lactate, CK-MB and troponin T in CO poisoning. Radiological evaluation should be used for excluding the reasons for a neurological disorder, not for diagnostic reasons. The first treatment option is $100 \%$ oxygen treatment for at least six hours. $\mathrm{HBO}$ treatment plan to reduce the half-live of $\mathrm{COHb}$ should be prepared considering prognostic factors; national protocols are required for the issue.

\section{Ethics}

Ethics Committee Approval: Following the approval of the Ethics Committee of Ministry of Health Okmeydanı Training and Research Hospital (approval date: 14/06/2016, decision no: 495), the patients admitted to emergency service with a pre-diagnosis of CO poisoning between the years 2014 and 2016 were screened retrospectively.

Informed Consent: Retrospective analysis.

Peer-review: Externally peer-reviewed.

\section{Authorship Contributions}

Surgical and Medical Practices: N.T., B.A., N.A., S.K., S.D.Ö., E.D., H.F., Y.I., T.M., Concept: N.T., B.A., Design: N.T., B.A., Data Collection or Processing: N.T., B.A., N.A., I.G., E.D., H.F., Y.I, Analysis or Interpretation: N.T., B.A., I.G., Literature Search: N.T., B.A., S.K., T.M., I.G., Writing: N.T., B.A., T.M. 
Conflict of Interest: The authors declare no conflict of interest.

Financial Disclosure: The authors declared that this study has received no financial support.

\section{REFERENCES}

1. Huysal K, Budak YU, Aydin U, Demirci H, Turk T, Karadag M. COHb Level and High-Sensitivity Cardiac Troponin T in 2012 in Bursa, Turkey: A Retrospective Single-Center Study. Iran Red Crescent Med J 2016;18:e27061.

2. Lang E, Qadri SM, Jilani K, Zelenak C, Lupescu A, Schleicher E, et al. Carbon monoxide-sensitive apoptotic death of erythrocytes. Basic Clin Pharmacol Toxicol 2012;111:348-55.

3. Moon JM, Shin HM, Chun JB. The value of initial lactate in patients with carbon monoxide intoxication: in the emergency department. Hum Exp Toxicol 2011;30:836-43.

4. T.C. Ministry of Health Turkish Public Health Authority (Environmental Health Department) Turkey Carbon Monoxide Poisoning Prevention Program and Action Plan (2015-2018). 1st Edition: Anıl Press. Kızılay / Ankara Available from: URL: http:/ www.cevresagligi.thsk.saglik.gov.tr

5. Onodera M, Fujino Y, Kikuchi S, Sato M, Mori K, Beppu T, et al. Utility of the Measurement of Carboxyhemoglobin Level at the Site of Acute Carbon Monoxide Poisoning in Rural Areas. Scientifica (Cairo) 2016;2016:6192369.

6. Bozeman WP, Myers RAM, Barish RA. Confirmation of the pulse oximetry gap in carbon monoxide poisoning. Ann Emerg Med 1997;30:608-11.

7. Bahcebasi T, Kandis H, Baltaci D, Kara IH. Factors affecting exhaled carbon monoxide levels in coffeehouses in the Western Black Sea region of Turkey. Toxicol Ind Health 2011;27:195-204.

8. Choi IS. Carbon monoxide poisoning: systemic manifestations and complications. J Kor Med Sci 2001;16:253-61.

9. Bernas S, Rzepeck J, Winnicka R, Kołaciński Z, Krakowiak A. Acute poisonings with carbon monoxide among the patients hospitalized in the Toxicology Unit during the period of 2006-2010. Przegl Lek 2012;69:415-9.

10. Ozyurt A, Karpuz D, Yucel A, Tosun MD, Kibar AE, Hallioglu O. Effects of Acute Carbon Monoxide Poisoning on ECG and Echocardiographic Parameters in Children. Cardiovasc Toxicol 2017;17:326-34.
11. Doğan NÖ, Savrun A, Levent S, Günaydın GP, Çelik GK, Akküçük H, et al. Can initial lactate levels predict the severity of unintentional carbon monoxide poisoning? Hum Exp Toxicol 2015;34:324-9.

12. Repplinger DJ, Slomer A, Nolan B, Su MK. Lactate and carbon monoxide poisoning: More questions regarding its prognostic role. Hum Exp Toxicol 2016;35:794.

13. Buckley NA, Juurlink DN, Isbister G, Bennett MH, Lavonas EJ. Hyperbaric oxygen for carbon monoxide poisoning. Cochrane Database Syst Rev 2011;CD002041.

14. Kandiş H, Katırcı Y, Çakır Z, Aslan \$̧, Uzkeser M, Bilir Ö. A Retrospective Analysis of Patients Admitted to the Emergency Service for Carbon Monoxide Intoxication. Eurasian J Emerg Med 2007;5:21-5.

15. Bleecker ML. Carbon monoxide intoxication. Handb Clin Neurol 2015;131:191-203.

16. Burney RE, Wu SC, Nemiroff MJ. Mass carbonmonoxide poisoning: Clinical effects and results of treatment in 184 victims. Ann Emerg Med 1982;11:394-9.

17. Hampson NB, Dunford RG, Kramer CC, Norkool DM. Selection criteria utilized for hyperbaric oxygen treatment of carbon monoxide poisoning. J Emerg Med 1995;13:227-31.

18. Weaver LK. Hyperbaric oxygen therapy for carbon monoxide poisoning. Undersea Hyperb Med 2014;41:339-54.

19. Pracyk JB, Stolp BW, Fife CE, Gray L, Piantadosi CA. Brain computerized tomography after hyperbaric oxygen theraphy for carbon monoxide poisoning. Undersea Hyperb Med 1995;22:1-7.

20. Tomaszewski CA, Thom SR. Use of hyperbaric oxygen in toxicology. Emerg Med Clin North Am 1994;12:437-59.

21. Mutluoglu M, Metin S, Ibrahim Arziman, Uzun G, Yildiz S. The use of hyperbaric oxygen therapy for carbon monoxide poisoning in Europe. Undersea Hyperb Med 2016;43:49-56.

22. Chan MY, Au TTs, Leung KS, Yan WW. Acute carbon monoxide poisoning in a regional hospital in Hong Kong: historical cohort study. Hong Kong Med J 2016;22:46-55.

23. Jüttner B, Tetzlaff $K$. Hyperbaric therapy and diving medicine hyperbaric therapy part 1:evidence-based emergency care. Anasthesiol Intensivmed Notfallmed Schmerzther 2015;50:618-25.

24. Tetzlaff K, Jüttner B. Hyperbaric therapy and diving medicinehyperbaric therapy part 2:adjuvant therapy. Anasthesiol Intensivmed Notfallmed Schmerzther 2015;50:628-35. 